\title{
Bridging Social Network Analysis and Judgment Aggregation
}

\author{
Silvano Colombo Tosatto and Marc van Zee \\ University of Luxembourg
}

\begin{abstract}
Judgment aggregation investigates the problem of how to aggregate several individuals' judgments on some logically connected propositions into a consistent collective judgment. The majority of work in judgment aggregation is devoted to studying impossibility results, but the relationship between the (social) dependencies that may exist between voters and the outcome of the voting process is traditionally not studied. In this paper, we use techniques from social network analysis to characterize the relations between the individuals participating in a judgment aggregation problem by analysing the similarity between their judgments in terms of social networks. We obtain a correspondence between a voting rule in judgment aggregation and a centrality measure from social network analysis and we motivate our claims by an empirical analysis. We also show how large social networks can be simplified by grouping individuals with the same voting behavior.
\end{abstract}

\section{Introduction}

Social choice theory studies the problem how to reach collective consent between a group of people in the area of economic theory. It includes among others voting theory, preference aggregation and judgment aggregation. Judgment aggregation is the most recent formal theory of social choice, which investigates how to aggregate individual judgments on logically related propositions to a group judgment on those propositions. Examples of groups that need to aggregate individual judgments are expert panels, legal courts, boards, and councils. The problem of aggregating judgments gained popularity in the last ten years, since it has been shown to be general in the sense that both voting theory and preference aggregation are subsumed by it [16]. The majority of work in judgment aggregation is devoted to studying impossibility results similar to the work in preference aggregation by Arrow $[1,15]$, leading to the development of a large number of aggregation rules such as majority outcome, premise-based aggregation, and conclusion-based aggregation [16]. These rules are all concerned with the general problem of selecting outputs that are consistent or compatible with individual judgments [11]. However, the relation between the (social) dependencies that may exist between the voters and the outcome of the voting scenario is traditionally not studied.

Arguably, there may exist (social) relationships between the individuals that can have an influence on their individual judgments, and consequently on the 
aggregated outcome. For instance, a subgroup of the individuals can be close friends and therefore vote alike, or an individual in the group may be a dominant person and thus may influence the voting behavior of other voters. A representation of the social structure of a judgment aggregation problem makes it possible to identify influential voters in the entire group or in a subgroup of voters. This information can be useful for different purposes. Firstly, it can be used to determine the outcome of the voting process, simply by looking at what voters have a central position in the voting process and deriving the outcome from these voters. Secondly, it can be used to detect cartels in voting scenarios. A cartel is a formal, explicit agreement among competing firms. It is a formal organization of producers and manufacturers that agree to fix prices, marketing, and production. Finally, the social dependencies may allow one to simplify the voting problem by reducing the number of voters to the most important ones.

It does not seem obvious to extract such information from a judgment aggregation scenario, merely by relying on the tools that judgment aggregation offers. However, we believe that a possible natural solution to this problem can be provided by using techniques from social network analysis (SNA) to derive dependencies between voters. SNA views social relationships in terms of graph theory, consisting of nodes (representing individuals within the network) and ties (which represent relationships between the individuals, such as friendship, kinship, organisational position, sexual relationships, etc.) [21,4]. These networks are often depicted in a social network diagram, where nodes are represented as points and ties are represented as lines. The centrality of vertices, or the identification of which vertices are more "central" than others, is a key issue in network analysis.

In this paper, we explore the possibility to apply SNA to judgment aggregation by systematically translating a judgment aggregation problem to a social network. This social network reflects the agreement between voters derived from their judgments on the issues in the judgment aggregation problem. We analyse this network using the degree centrality measure, which is arguably the most well-known measure of node centrality from SNA. We formally prove an equivalence between the "average voter" voting rule in judgment aggregation and the degree centrality measure, showing that the social network can be used as an instrument to decide on a consistent and compatible outcome in the voting process. We motivate our claim with an experimental analysis, indicating that by varying the parameters of the centrality measure, we are able to fine-tune the outcome of the voting process. Finally, we show that large networks with many voters and few issues can be simplified significantly by clustering individuals that vote the same.

The paper is organised as follows: We start by discussing related work in Section 2. In Section 3 we introduce the basic notions of judgment aggregation and two voting rules, and we introduce basic terminology from social network analysis in Section 4. In Section 5 we show how we can systematically obtain a social network from a judgment aggregation problem using simple matrix operations. We use this method in Section 6, where we show a correspondence between 
a centrality measure on the graph and a voting rule in judgment aggregation. Empirical results are discussed in Section 7 and we show how to simplify the social networks in Section 8.

\section{Related Work}

There is substantial research in social science showing that social dependencies exist between voters and that this can have an influence on the outcome of the voting process. For instance, Gerber et al. [9] performed a large-scale field experiment involving several hundred thousand registered voters, demonstrating the profound importance of social pressure as an inducement to political participation. Nickerson [19] performs two field experiments showing that within households, $60 \%$ of the propensity to vote is passed onto the other member of the household. This suggests a mechanism by which civic participation norms are adopted and couples grow more similar over time. Kenny [12] uses survey responses from the 1984 South Bend study to model the relationship between political discussion partners. Again, the evidence indicates that certain types of both individually based and socially based participation are affected by those in the immediate social environment.

Possibly caused by the recent popularity of online social networks such as Facebook, Twitter, LinkIn, Pinterest, and others, most recent research combining social choice theory with social network analysis pursues in the opposite direction from ours. Social networks are taken as the starting point and one investigates to what extent fair and consistent voting can be implemented on such networks. For instance, both Salehi-Abari and Boutilier [22] as well as Boldi et al. [2] study how members of a social network derive utility based on both their own preferences and the satisfaction of their neighbors. Here, users can only express their preferences for one among the people they are explicitly connected with, and this preferences can be propagated transitively. Both Lerman and Galstyan [13] and Lerman and Ghosh [14] study the role of social networks in promoting content on Digg, a social news aggregator that allows users to submit links to and vote on news stories. Their results suggest that pattern of the spread of interest in a story on the network is indicative of how popular the story will become.

There is significantly less work trying to obtain social networks from social choice problems. Endriss and Grandi [5] investigate the problem of graph aggregation, where individuals do not give a judgment over alternatives, but instead provide a directed graph over a common set of vertices. Judgment aggregation reduces then to computing a single graph that best represents the information inherent in this profile of individual graphs. This is considerably different from our work, since we obtain a graph from the dependencies between voters, assuming that voters give a judgment over alternatives. 


\section{Judgment Aggregation}

In this section we recall the framework of judgment aggregation $[16,24]$. The problem is formulated as binary aggregation with integrity constraints, which is equivalent to judgment aggregation when the individual judgments are complete and consistent [10]. We also define several voting rules that we use throughout the paper.

\subsection{Basic Definitions}

A judgment aggregation problem consists of a set of individuals having to aggregate their preferences over a set of issues. The preferences of each individual are expressed by saying either yes or no for each of the issues proposed.

Let $\mathcal{N}=\{1,2, \ldots, n\}$ be a finite set of individuals, and let $\mathcal{I}=\{1,2, \ldots, m\}$ be a finite set of issues. We want to model collective decision making problems where the group of individuals $\mathcal{N}$ have to jointly decide for which issues in $\mathcal{I}$ to choose "yes" and for which to choose "no". A ballot $B \in\{0,1\}^{m}$ associates either 0 ("no") or 1 ("yes") with each issue in $\mathcal{I}$. We write $B_{j}$ for the $j$ th element of $B$. Thus, $B_{j}=1$ denotes that the individual has accepted the $j$ th issue, and $B_{j}=0$ denotes that the individual has rejected it.

In general, not every possible ballot might be a feasible or rational choice. For instance, if the issues are tasks that are to be executed by a group of people, then a task constraint might mean that deciding to execute certain tasks makes it impossible to execute other tasks.

Formally, let $P S=\left\{p_{1}, \ldots, p_{m}\right\}$ be a set of propositional symbols, one for each issue $\mathcal{I}$. An integrity constraint is a formula $I C \in \mathcal{L}_{P S}$, where $\mathcal{L}_{p s}$ is obtained from $P S$ by closing under the standard propositional connectives. Let $\operatorname{Mod}(I C) \subseteq\{0,1\}^{m}$ denote the set of models of IC, i.e. the set of rational ballots satisfying IC.

A profile is a vector of rational ballots $\mathbf{B}=\left(B_{1}, \ldots, B_{n}\right) \in \operatorname{Mod}(\operatorname{IC})^{n}$, containing one ballot for each individual. We write $B_{i j}$ to denote the $i$ th individual's choice about the $j$ th issue, i.e. the $j$ th choice of ballot $B_{i}$. Since ballots are vectors themselves, we can consider $\mathbf{B}$ as a matrix of size $n \times m$. The support of a profile $\mathbf{B}=\left(B_{1}, \ldots, B_{n}\right)$ is the set of all ballots that occur at least once within B:

$$
\operatorname{SupP}(\mathbf{B})=\left\{B_{1}\right\} \cup \ldots \cup\left\{B_{n}\right\} .
$$

A voting rule $F:\{0,1\}^{m \times n} \rightarrow 2^{\{0,1\}^{m}}$ is a function that maps each profile $\mathbf{B}$ to a set of ballots. This means that an aggregation rule can have one or multiple outcomes, also called an irresolute voting rule. A voting rule is called collectively rational when all outcomes satisfy the integrity constraints.

One of the most well-known voting rules is the (weak) majority rule, which accepts an issue if a weak majority accepts it:

$$
\operatorname{Maj}(\mathbf{B})_{j}=1 \text { iff }\left|\left\{i \in \mathcal{N} \mid \mathbf{B}_{i j}=1\right\}\right| \geq\left\lceil\frac{n}{2}\right\rceil .
$$


Example 1. Suppose the following judgment aggregation scenario consisting of six individuals $(a, b, c, d, e, f)$ voting on an agenda composed of four issues $(p, q, r, z)$. The agenda is subject to the following integrity constraint: $I C=(p \wedge q \wedge r) \Leftrightarrow z$. The majority outcome is depicted in the last row.

\begin{tabular}{r|cccc} 
Issue: & $\mathbf{p}$ & $\mathbf{q}$ & $\mathbf{r}$ & $\mathbf{z}$ \\
\hline $\mathrm{a}$ & 0 & 1 & 1 & 0 \\
$\mathrm{~b}$ & 1 & 0 & 0 & 0 \\
$\mathrm{c}$ & 1 & 1 & 1 & 1 \\
$\mathrm{~d}$ & 1 & 0 & 0 & 0 \\
$\mathrm{e}$ & 1 & 0 & 1 & 0 \\
$\mathrm{f}$ & 0 & 0 & 1 & 0 \\
\hline Maj & 1 & 0 & 1 & 0
\end{tabular}

The Hamming distance between two ballots $B=\left(B_{1}, \ldots, B_{m}\right)$ and $B^{\prime}=$ $\left(B_{1}^{\prime}, \ldots, B_{m}^{\prime}\right)$ is defined as the sum of the amount of issues on which they differ:

$$
H\left(B, B^{\prime}\right)=\left|\left\{j \in \mathcal{I} \mid B_{j} \neq B_{j}^{\prime}\right\}\right|
$$

For example, $H((1,0,0),(1,1,1))=2$. The Hamming distance between a ballot $B$ and a profile $\mathbf{B}$ is the sum of the Hamming distances between $B$ and the ballots in $\mathbf{B}$ :

$$
\mathcal{H}(B, \mathbf{B})=\sum_{i \in \mathcal{N}} H\left(B, B_{i}\right)
$$

\subsection{The Average Voter Rule}

Endriss and Grandi [6] recently proposed the average voter rule, which reduces the space of the possible outcomes to the ballots proposed by the voters. In this way, the consistency of the outcome of the voting process is guaranteed, given that all voters vote consistently. It was later shown by Grandi and Pigozzi [11] that this rule satisfies several desirable properties.

Definition 1 (AVR). The average voter rule (AVR) is the voting rule that selects those individual ballots that minimise the Hamming distance to the profile:

$$
A V R(\boldsymbol{B})=\underset{B \in \operatorname{Supp}(B)}{\operatorname{argmin}} \mathcal{H}(B, \boldsymbol{B})
$$

\section{Social Network Analysis}

A social network usually is represented as a graph. The vertices are the individuals, and the edges represent the social connections. In this paper, we consider the symmetric case where social networks are represented by undirected graphs. An edge which joins a vertex to itself is called a loop. The number of edges that are incident to a vertex is called the degree of a vertex. The neighborhood of a vertex $v$ is the set of all vertices adjacent to $v$. 
We denote a weighted network (or weighted graph) with $G=(V, E, W)$ with the vertex set $V(G)=\left\{v_{1}, \ldots, v_{n}\right\}$, edge set $E$, and weight matrix $W$, where each edge $e=\left(v_{i}, v_{j}\right)$ is labeled with a weight $w_{i j}$. We assume that if two vertices are not connected, then there exists an edge of weight 0 connecting them. Since we only consider undirected networks, $w_{i j}=w_{j i}$. We define the sum-weight $s_{i}$ of a vertex $v_{i}$ with $s_{i}=\sum_{j=1}^{n} w_{i j}=\sum_{u \in N\left(v_{i}\right)} w_{v_{i} u}$, where $N\left(v_{i}\right)$ is the neighborhood of $v_{i}$. We denote the degree $k_{i}$ of a vertex $v_{i}$ with $k_{i}=\left|N\left(v_{i}\right)\right|$, i.e. $k_{i}$ denotes the number of neighbors of $v_{i}$.

The centrality of vertices, identifying which vertices are more "central" than others, has been a key issue in network analysis. Freeman [8] originally formalized three different measures of vertex centrality: degree, closeness, and betweenness. In this paper, we will only consider the degree centrality. Degree is the number of vertices that a focal vertex is connected to, and measures the local involvement of the vertex in the network. This measure is originally formalised for binary graphs [8], but we will consider recent proposal [20] that uses a tuning parameter $\alpha$ to control the relative importance of number of edges compared to the weights on the edges.

The degree centrality measure is defined as the product of the number of vertices that a focal vertex is connected to, and the average weight to these vertices adjusted by the tuning parameter. The degree centrality for a vertex $i$ is computed as follows:

$C_{D}^{W \alpha}(i)=k_{i} \times\left(\frac{s_{i}}{k_{i}}\right)^{\alpha}=k_{i}^{(1-\alpha)} \times s_{i}^{\alpha}$

where $W$ is the weight matrix of graph, $\alpha$ is a positive tuning parameter, $k_{i}$ is the size of the neighborhood of vertex $i$ and $s_{i}$ the sum of the weights of the incident edges. If $\alpha$ is between 0 and 1, then having a high degree is favorable over weights, whereas if it is set above 1, a low degree is favorable over weights. In Section 6 we elaborate on different levels of $\alpha$ for degree centrality.

\section{Towards a Social Network}

In this section we will bridge the two problem domains that we introduced above using a technique introduced in social theory by Breiger [3]. This technique is originally used to analyse membership of people to groups, however we use it to represent agreement between voters.

\subsection{Matrix Translation}

We use the following transformations to obtain a social network from a voting profile.

Definition 2 (Similarity matrix). Given a profile matrix $\boldsymbol{B}$. The similarity matrix $\mathcal{B}$ is obtained from $\boldsymbol{B}$ as follows:

$$
\mathcal{B}_{i j}= \begin{cases}1 & \text { if } \boldsymbol{B}_{i j}=1 \\ -1 & \text { if } \boldsymbol{B}_{i j}=0\end{cases}
$$


Definition 3 (Voter-to-voter matrix). Given a similarity matrix $\mathcal{B}$ of size $n \times m$ and $V^{*}=\mathcal{B}\left(\mathcal{B}^{T}\right)$, where multiplication is ordinary (inner product) matrix multiplication. The voter-to-voter matrix $V$ of $V^{*}$ is constructed as follows:

$$
V_{i j}=\frac{V_{i j}^{*}+m}{2}
$$

The following theorem states the main result of this section, showing that the voter-to-voter matrix counts the equal elements between each two rows of the original profile matrix.

Theorem 1. Let $\boldsymbol{B}$ be a profile matrix of size $n \times m, \mathcal{B}$ the similarity matrix of $\boldsymbol{B}$, and $V$ the corresponding voter-to-voter matrix of $\mathcal{B} . V_{i j}$ contains the amount of equal elements in row $i$ and $j$ of $\boldsymbol{B}$, i.e.:

$$
V_{i j}=\left|\left\{\boldsymbol{B}_{i k} \mid \boldsymbol{B}_{i k}=\boldsymbol{B}_{j k}, 1 \leq k \leq m\right\}\right|
$$

Thus, $V_{i j}$ denotes the number of times that both voters $i$ and $j$ voted "yes" or they both voted "no" for the same issue.

Example 2 (Continued). We can translate the matrix $\mathbf{B}$ of Example 1 that corresponds to this voting profile to a similarity matrix (Figure 1a). Next, we calculate $V^{*}$ and obtain the the voter-to-voter matrix $V$ after normalising the result (Figure 1b).

\begin{tabular}{c|rrrr} 
& $\mathrm{p}$ & $\mathrm{q}$ & $\mathrm{r}$ & $\mathrm{z}$ \\
\hline $\mathrm{a}$ & -1 & 1 & 1 & -1 \\
$\mathrm{~b}$ & 1 & -1 & -1 & -1 \\
$\mathrm{c}$ & 1 & 1 & 1 & 1 \\
$\mathrm{~d}$ & 1 & -1 & -1 & -1 \\
$\mathrm{e}$ & 1 & -1 & 1 & -1 \\
$\mathrm{f}$ & -1 & -1 & 1 & -1
\end{tabular}

(a) Voting Profile B

\begin{tabular}{c|cccccc} 
& $\mathrm{a}$ & $\mathrm{b}$ & $\mathrm{c}$ & $\mathrm{d}$ & $\mathrm{e}$ & $\mathrm{f}$ \\
\hline $\mathrm{a}$ & 4 & 1 & 2 & 1 & 2 & 3 \\
$\mathrm{~b}$ & 1 & 4 & 1 & 4 & 3 & 2 \\
$\mathrm{c}$ & 2 & 1 & 4 & 1 & 2 & 1 \\
$\mathrm{~d}$ & 1 & 4 & 1 & 4 & 3 & 2 \\
$\mathrm{e}$ & 2 & 3 & 2 & 3 & 4 & 3 \\
$\mathrm{f}$ & 3 & 2 & 1 & 2 & 3 & 4
\end{tabular}

(b) Voter-to-voter matrix (V)

Fig. 1. Transforming the voting profile to a voter-to-voter matrix.

The voter-to-voter matrix is symmetric with respect to its main diagonal: If some voter $i$ agrees with a voter $j$ on some issues, then $j$ agrees with $i$ on the same issues as well. This implies reflexivity: a voter always agrees with itself over every issue, and similarly for any issue. Therefore, the main diagonal of the voter matrix is always equal to the number of issues.

\subsection{Relational Graphs}

The voter-to-voter matrix $V$ can be represented as an undirected, weighted graph. In such a graph, a voter is represented by a node, and an edge represents the agreement between two voters. Formally, an edge $(i, j)$ connects two 
vertices $i$ and $j$ if the matrix entry $V_{i j}$ has a value larger than 0 . We denote the obtained graphs with $G_{V}=\left(V_{V}, E_{V}, W_{V}\right)$. Notice that the matrix $V$ is equivalent to the weight matrix of the corresponding graph, i.e. $V=W_{V}$. We call the graph $G_{V}$ as the voter graph.

Example 3 (Continued). Figure 2 shows the voting graph resulting from the voter-to-voter matrix depicted in Figure $1 \mathrm{~b}$. We can see that the strongest connection is between the individuals $b$ and $d$, representing the fact that their ballots are equivalent. Differently, $c$ can be considered an outlier due to its weak connections with the other individuals. Note that for the sake of readability, the edges with a weight of 1 have not been labeled in Figure 2 and reflexive edges have been omitted.

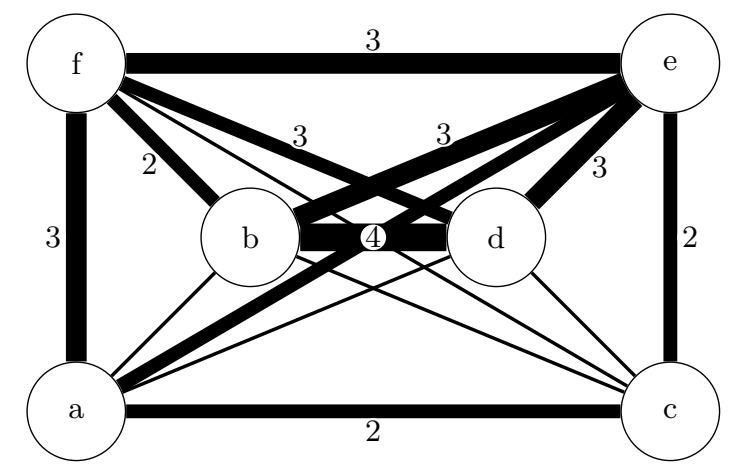

Fig. 2. Voter graph $\left(G_{V}\right)$

The voter-to-voter graph expresses the agreement between the individuals through the weighted edges. In the following section we show that the individuals in the voter graph that are most central according to the degree centrality measure corresponds to the voters that are selected by the average voter rule in the judgment aggregation profile.

\section{Theoretical Analysis}

We start out with a straightforward equivalence between the Hamming distance between two voters and the edge that connects the two voters in the corresponding voting graph.

Lemma 1. The Hamming distance between two ballots $B_{i}$ and $B_{j}$ is equal to $m-w_{i j}$ in the corresponding voter graph $G_{V}$, i.e. $H\left(B_{i}, B_{j}\right)=m-w_{i j}$.

We use this lemma to obtain an equivalence between the Hamming distance to a profile and the total weight of the corresponding node in the voter graph. 
Lemma 2. The Hamming distance between a ballot $B_{i}$ and a profile $\boldsymbol{B}$ is equal to $m n-s_{i}$, where $s_{i}$ is the sum of the weights of the incident edges of vertex $i$ in the voter graph constructed from $\boldsymbol{B}$ :

$$
H\left(B_{i}, \boldsymbol{B}\right)=m n-s_{i}
$$

Example 4 (Continued). In Example 1, we have $H(a, b)=3$ and $H(a, \mathbf{B})=11$. In the corresponding graph in Figure 2 we have that $w_{a b}=1$ and thus $m-$ $w_{a b}=4-1=3$, which corresponds to the Hamming distance between $a$ and $b$. Moreover, $s_{a}=13$ (including the reflexive weight of 4 ), so $m n-s_{a}=24-13=11$, which corresponds to the Hamming distance between $a$ and the profile $\mathbf{B}$.

Since the average voter rule selects the voter that minimises the distance with the profile, we can obtain the following equivalence:

Lemma 3. The average voter rule (AVR) (Definition 1) selects the voters corresponding to the maximum total weight vertices in the voter graph, i.e.:

$$
A V R(\boldsymbol{B})=\underset{i \in V_{V}}{\operatorname{argmax}} s_{i} .
$$

Next, we obtain that the average voter rule corresponds to the node with the highest degree centrality when the tuning parameter $\alpha=1$ :

Theorem 2. The AVR selects those individual ballots that have the maximal degree centrality value when $\alpha=1$. Suppose $\alpha=1$ :

$$
A V R(\boldsymbol{B})=\underset{i \in V_{V}}{\operatorname{argmax}} C_{D}^{W \alpha}(i)
$$

Proof. Follows directly from Eq.(1) and Lemma 3

Example 5. Consider the voting profile in Figure 3a, where a set of four voters have to decide on five issues. We can see in the bottom part of the table that the average voter rule corresponds to the set of individuals $\{a, c, d\}$. The voter graph of this voting profile is shown in Figure 3b. Recall that the degree centrality score for the nodes when $\alpha=1$ can be calculated by summing up the weights of all incident edges. Thus, the value of node $a, c$, and $d$ are all 6 while the value of node $b$ is 4 . Therefore, the set of voters selected using the degree centrality measure is $\{a, c, d\}$ as well, which is in line with Theorem 2 .

\section{Empirical Analysis}

In this section we analyse the effect of varying the tuning parameter $\alpha$ in the degree centrality measure by comparing the outcomes with those of the average voter rule, taking the majority voting rule as our base measure. We first provide an intuitive discussion on the effect of varying the tuning parameter, followed by an empirical analysis. 


\begin{tabular}{c|ccccc} 
& 1 & 2 & 3 & 4 & 5 \\
\hline $\mathrm{a}$ & 0 & 1 & 1 & 1 & 1 \\
$\mathrm{~b}$ & 1 & 0 & 0 & 0 & 0 \\
$\mathrm{c}$ & 0 & 1 & 1 & 0 & 0 \\
$\mathrm{~d}$ & 0 & 0 & 0 & 1 & 1 \\
\hline AVR: & 0 & 1 & 1 & 1 & 1 \\
& 0 & 1 & 1 & 0 & 0 \\
& 0 & 0 & 0 & 1 & 1
\end{tabular}

(a) Profile

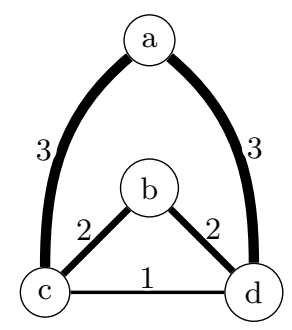

(b) Voter graph

Fig. 3. Example judgment aggregation profile with voter-to-voter graph

\subsection{Varying the tuning parameter}

Reconsider the voting scenario together with the majority outcomes and the average voter outcomes of Figure 3. The outcome of the degree centrality for varying $\alpha$ are depicted in Figure 4. As can be seen from the table, for $\alpha=1$, the degree centrality measure corresponds to the $s_{i}$ measure, which measures the sum of the weights of the edges connected to that node. Therefore the nodes $a, c$ and $d$ are all chosen as the average voter because of their greater degree centrality measure. When $\alpha<1$ the amount of edges play are larger role and only $c$ and $d$ are chosen as the most central because of their three connected edges against the two of nodes $a$ and $b$. Contrast this with $\alpha>1$, when the weight of the edges play the prominent role in deciding the most central node. In this case $a$ is picked as the most central node by having the edges with the largest weights connected to it. This analysis suggests that in some cases, by using different values for the tuning parameter $\alpha$ to compute the most central node in a graph, it is possible to obtain a more fine-grained voting rule than the result of the average voter rule.

The outcomes obtained using the degree centrality measure can be compared with the vector of "average votes" $\left(\frac{1}{4}, \frac{2}{4}, \frac{2}{4}, \frac{2}{4}, \frac{2}{4}\right)$, showing for each issue the proportion of voters who chose 1 rather than 0 . We can see that only the first issue is uncontroversial, while the no unique decision on the other issues is possible. Having multiple available outcomes is not uncommon for voting rules such as the majority rule and AVR. However, in these cases fine tuning the $\alpha$ parameter may lead to more resolute outcomes by exploiting the structure of the voter graph.

\subsection{Experimental Setup}

The setup of the empirical analysis performed ${ }^{1}$ consists of a judgment aggregation problem with 100 voters and 4 issues, with no integrity constraints. We have chosen for relatively many voters because the degree centrality measure is based

\footnotetext{
${ }^{1}$ The experiment has been coded in Java and can be found on the web: http://icr.uni.lu/marc/code/socinfo2014/src.zip
} 


\begin{tabular}{llllll} 
Vertex & $s_{i}$ & \multicolumn{6}{c}{$C_{D}^{W \alpha}$ when $\alpha=$} \\
\cline { 3 - 6 } & & 0 & 0.5 & 1 & 1.5 \\
\hline a & $\mathbf{6}$ & 2 & 3.46 & $\mathbf{6}$ & $\mathbf{1 0 . 3 9}$ \\
b & 4 & 2 & 2.83 & 4 & 5.66 \\
c & $\mathbf{6}$ & $\mathbf{3}$ & $\mathbf{4 . 2 4}$ & $\mathbf{6}$ & 8.48 \\
d & $\mathbf{6}$ & $\mathbf{3}$ & $\mathbf{4 . 2 4}$ & $\mathbf{6}$ & 8.48
\end{tabular}

Fig. 4. Degree centrality scores when different values of $\alpha$ are used.

on graph theory whereby these measures are more effective on large graphs due to the more dependencies and similarities between the individuals. We leave out the integrity constraints since logical constraints on the issues are not the focus of our work. The votes are generated pseudo-randomly such that all votes are complete, meaning that each voters votes either "yes" or "no" for each issue.

In order to compare the different measures we use the majority rule as the base measure. The majority rule is generally considered to be the most wellknown voting rule, and is most likely also one of the most used rules. We compare the outcome of the average voter rule and the degree centrality measure for different values of $\alpha$ with the base measure by computing the Hamming Distance.

The experiment is reiterated 5000 times for each value of the tuning parameter $\alpha$. If a measure produces multiple outcomes, we measure the distance to the base measure for each result. All these distances are stored in a list $L_{M}$ for each measure $M$. For each value of the tuning parameter we use $L_{M}$ to compute the mean, the standard deviation $\sigma$ and the average number of outcomes per benchmark $O_{a v g}$, i.e. $O_{a v g}=\frac{\left|L_{M}\right|}{5000}$ for the measure $M$. The value $O_{a v g}$ can be seen as a measure for resoluteness: The closer this number is to 1 , the more resolute the voting rule is, which means that the number of outcomes is effectively smaller.

\subsection{Results and Analysis}

Figure 5 shows the results of the experiment. From the figure it can be seen that, as shown in Section 6, the average voter rule corresponds to the degree centrality measure when the tuning parameter $\alpha=1$. When the value of $\alpha$ increases from 1 to 3 , the average distance to the base measure slowly increases, meaning that the result of the degree centrality measure is further away from the majority based rule. On the other hand, the average number of outcomes also decreases, which seems to suggest that while the voting rule becomes more resolute (i.e. results in less outcomes) when $\alpha$ increases, it also becomes less precise. The results for $\alpha=0$ are somewhat surprising, since the average distance to the base measure is very high compared to the average distance of the average voter rule, and the average number of outcomes is very large as well. A possible explanation for this deviation may have to do with the density of the graph. When $\alpha$ is 0 , the weights on the edges is completely disregarded and the degree centrality value of a node is solely determined by the number of other nodes connected to 
it (see Equation 1). The networks that we obtain are usually rather dense, so it seems that selecting an outcome merely based on the number of ties is not precise enough, which might explain the large number of outcomes for $\alpha=0$. For $\alpha$ values smaller than 0 or larger than 3 , the results remained more or less constant.

\begin{tabular}{lc|l|l|l} 
Voting rule & $\alpha$ & mean & $\sigma$ & $O_{\text {avg }}$ \\
\hline MRV & - & 0.06 & 0.24 & 1.9 \\
\hline Degree & 0.0 & 1.16 & 0.88 & 7.2 \\
& 0.5 & 0.07 & 0.26 & 1.88 \\
& 1.0 & 0.06 & 0.24 & 1.9 \\
& 1.5 & 0.07 & 0.24 & 1.83 \\
& 2.0 & 0.07 & 0.26 & 1.83 \\
& 2.5 & 0.08 & 0.28 & 1.81 \\
& 3.0 & 0.10 & 0.33 & 1.77
\end{tabular}

Fig. 5. Benchmarking results showing Hamming distances from majority based rule

\section{Simplifying the Social Network}

As we mentioned previously, it can be the case that a judgment aggregation problem features a big set of voters having to decide over a small set of issues. In this case it is inevitable that many of the voters involved in the voting process will have identical votes. Consider for instance a group of 100 voters that has to decide over 4 issues. The number of possible voting profiles (assuming no integrity constraints) is $2^{4}=16$, meaning that there will be at least 84 nonidentical voters, so at most $16 \%$ of the voting profiles are unique.

A group of individuals voting the same way is represented in the voter graph as a strongly connected component of the graph where each of the connections among the nodes in the component has weight equal to the amount of issues in the voting scenario. In addition to the connections among the tightly connected components, the nodes are also connected to other nodes using edges with variable weights depending on the amount of agreement between the voters represented by the nodes as it is shown in Figure 6 .

By having a high number of nodes in the graph it is necessary to calculate the degree centrality measure of each one of them in order to decide the most central one(s). Moreover the representation of the voter graph would be cluttered by all the edges being the graph almost completely connected. Additionally, when considering the most central nodes in such graphs, all nodes belonging to the same strongly connected component have the same degree centrality value, hence if one of them is the most central, then each of them is.

Both problems, the cluttered graph and redundant calculations of the degree centrality, can be solved by reducing the amount of node represented in the graph itself. Because the nodes that belong to the same strongly connected 


\begin{tabular}{c|ccc} 
& 1 & 2 & 3 \\
\hline $\mathrm{a}$ & 0 & 0 & 1 \\
$\mathrm{~b}$ & 1 & 1 & 0 \\
$\mathrm{c}$ & 1 & 1 & 0 \\
$\mathrm{~d}$ & 0 & 0 & 0 \\
$\mathrm{e}$ & 0 & 0 & 0 \\
$\mathrm{f}$ & 1 & 1 & 0
\end{tabular}

(a) Profile

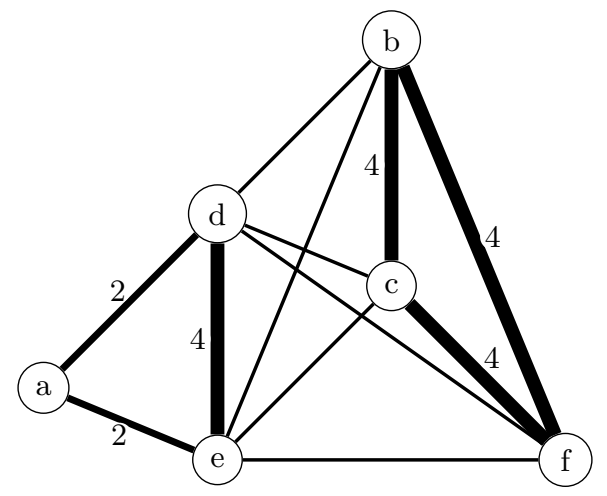

(b) Voter Graph

Fig. 6. Non-Simplified Translation

components have the same properties in term of centrality, we can represent each strongly connected component as a single node in the graph and connect it to the other strongly connected components (also represented by a single node) using edges weighted according to the agreement. To keep track of the size of the strongly connected components reduced to nodes, a weight equivalent to the cardinality of the component is associated to the node. The simplified voting graph of Figure 6b is shown in Figure 7.

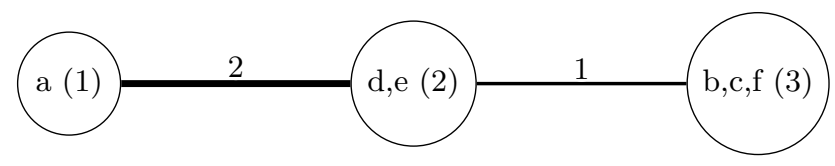

Fig. 7. Simplified Voter Graph

The degree centrality on the simplified graph can be calculated for each of the nodes using the following equations, which produces a result equivalent to the one that would have been obtained by calculating it on a node belonging to the strongly connected component in the non simplified graph. The two equations allow to compute in the simplified graph the size of the neighbourhood $k_{i}$ and the size of sum of the weights of the connected edges $s_{i}$.

$$
\begin{aligned}
& s_{n}=i \cdot\left(c_{n}-1\right)+\sum_{(n, m) \in E} w_{n m} \cdot c_{m} \\
& k_{n}=\left(c_{n}-1\right)+\sum_{(n, m) \in E} c_{m}
\end{aligned}
$$


Where $c_{i}$ is the size of the strongly connected component to which node $i$ belongs, $i$ is the number of issues, and $w_{n m}$ is the weight of the edge between $n$ and another node $m$ in the voter graph.

\section{Conclusions and Future Work}

In the present paper we show that deciding the average voter in a judgment aggregation problem corresponds to selecting the most central voter in a social network where the strength of the ties in the network follows from similar voting behavior of two individuals in the judgment aggregation problem.

To the best of our knowledge, this is the first attempt to correlate the two areas by showing that by remodelling the problems, classic techniques as the centrality measure used by Breiger [3] to analyse people membership to groups, is comparable to use the average voter rule, proposed by Grandi and Pigozzi [11], to solve a judgment aggregation problem.

The connection between the two fields shown in this work hints that some of the techniques used in one of the areas could be indeed adapted and reused in the other to solve some of the problems. As we show in our empirical analysis, by varying the tuning parameter $\alpha$ used to compute the centrality measure, the results obtained change. As discussed the parameter $\alpha$ switches the emphasis between the weights and the number of edges connecting the nodes, hence using a different tuning parameter than $\alpha=1$ already corresponds in some sense to a different voting rule, however whether these new rules can be useful is still to be decided.

Additionally, we propose a way to simplify a tightly connected graph where some strongly connected components are present. By collapsing the strongly connected components in a single node, we avoid to represent a cluttered and unreadable graph in addition to have to calculate the degree centrality measures for the collapsed nodes instead for the whole strongly connected component. For future work, we would like to compare this method against other graph sparsification methods such as $[23,7,18,17]$ to find out whether our approach can be optimized or extended. For instance, a more general treatment of this network simplification technique might refer to community detection, which is not restricted to cliques with the same weight but can define groups of nodes in other ways. ${ }^{2}$ Reducing the number of ties will also be useful because SNA methods are often conceived with sparse graphs in mind, while our approach often produces very dense graphs. In addition, the impact of the tuning parameter $\alpha$ seems to be related to these missing edges only and requires future study.

Lastly, consistency is one of the main objects of study in judgment aggregation. The fact that the proposed framework does not consider constraints seems to represent a significant limitation. In particular it does not seem obvious how the connection between traditional social network analysis (SNA) measures and voting rules can be maintained. We leave this to future studies.

\footnotetext{
${ }^{2}$ This was suggested by an anonymous reviewer
} 


\section{Acknowledgments}

Silvano Colombo Tosatto and Marc van Zee are supported by the National Research Fund, Luxembourg.

\section{References}

1. Kenneth J. Arrow. Social Choice and Individual Values. John Wiley and Sons, New York, NY, 1951.

2. Paolo Boldi, Francesco Bonchi, Carlos Castillo, and Sebastiano Vigna. Voting in social networks. In David Wai-Lok Cheung, Il-Yeol Song, Wesley W. Chu, Xiaohua $\mathrm{Hu}$, and Jimmy J. Lin, editors, CIKM, pages 777-786. ACM, 2009.

3. RL Breiger. The duality of persons and groups. Social forces, 1974.

4. Alessia D'Andrea, Fernando Ferri, and Patrizia Grifoni. An overview of methods for virtual social networks analysis. In Computational Social Network Analysis, Computer Communications and Networks, chapter 1, pages 3-25. Springer London, London, 2010.

5. Ulle Endriss and Umberto Grandi. Graph aggregation. In Proceedings of the 4th International Workshop on Computational Social Choice (COMSOC-2012), September 2012.

6. Ulle Endriss and Umberto Grandi. Binary aggregation by selection of the most representative voter. In Proceedings of the 7th Multidisciplinary Workshop on Advances in Preference Handling, August 2013.

7. Nicholas J Foti, James M Hughes, and Daniel N Rockmore. Nonparametric sparsification of complex multiscale networks. PloS one, 6(2):e16431, 2011.

8. Linton C. Freeman. Centrality in social networks conceptual clarification. Social Networks, page 215, 1978.

9. Alan S. Gerber, Donald P. Green, and Christopher W. Larimer. Social pressure and voter turnout: Evidence from a large-scale field experiment. American Political Science Review, 102:33-48, 22008.

10. Umberto Grandi and Ulle Endriss. Binary aggregation with integrity constraints. In Proceedings of the 22nd International Joint Conference on Artificial Intelligence, July 2011.

11. Umberto Grandi and Gabriella Pigozzi. On compatible multi-issue group decisions. In Proceedings of the 10th Conference on Logic and the Foundations of Game and Decision Theory, 2012.

12. Christopher B Kenny. Political participation and effects from the social environment. American Journal of Political Science, pages 259-267, 1992.

13. Kristina Lerman and Aram Galstyan. Analysis of social voting patterns on digg. In Proceedings of the First Workshop on Online Social Networks, WOSN '08, pages 7-12, New York, NY, USA, 2008. ACM.

14. Kristina Lerman and Rumi Ghosh. Information contagion: an empirical study of the spread of news on digg and twitter social networks. CoRR, abs/1003.2664, 2010 .

15. C. List and C. Puppe. Judgment aggregation: A survey. Handbook of Rational and Social Choice, 2009.

16. Christian List and Ben Polak. Introduction to judgment aggregation. Journal of Economic Theory, 145(2):441-466, March 2010. 
17. PJ Macdonald, E Almaas, and A-L Barabási. Minimum spanning trees of weighted scale-free networks. EPL (Europhysics Letters), 72(2):308, 2005.

18. Michael Mathioudakis, Francesco Bonchi, Carlos Castillo, Aristides Gionis, and Antti Ukkonen. Sparsification of influence networks. In Proceedings of the 17th ACM SIGKDD international conference on Knowledge discovery and data mining, pages 529-537. ACM, 2011.

19. David W Nickerson. Is voting contagious? evidence from two field experiments. American Political Science Review, 102(01):49-57, 2008.

20. Tore Opsahl, Filip Agneessens, and John Skvoretz. Node centrality in weighted networks: Generalizing degree and shortest paths. Social Networks, 32(3):245-251, July 2010.

21. C.A.R. Pinheiro. Social Network Analysis in Telecommunications. Wiley and SAS Business Series. Wiley, 2011.

22. Amirali Salehi-Abari and Craig Boutilier. Empathetic social choice on social networks. In Fourth International Workshop on Computational Social Choice, 2012.

23. M. ngeles Serrano, Marin Bogu, and Alessandro Vespignani. Extracting the multiscale backbone of complex weighted networks. Proceedings of the National Academy of Sciences, 106(16):6483-6488, 2009.

24. M Slavkovik. Judgment aggregation for multiagent systems. $\mathrm{PhD}$ thesis, Univerity of Luxembourg, 2012.

\section{A Appendix: Proofs}

Theorem 1. Let $\boldsymbol{B}$ be a profile matrix of size $n \times m, \mathcal{B}$ the similarity matrix of $\boldsymbol{B}$, and $O$ the corresponding normalised matrix of $\mathcal{B} . O_{i j}$ contains the amount of equal elements in row $i$ and $j$ of $\boldsymbol{B}$, i.e.:

$$
O_{i j}=\left|\left\{\boldsymbol{B}_{i k} \mid \boldsymbol{B}_{i k}=\boldsymbol{B}_{j k}, 1 \leq k \leq m\right\}\right|
$$

Proof. We prove this theorem directly.

1. Suppose arbitrary rows $\mathbf{B}_{i}, \mathbf{B}_{j}$ of some profile matrix $\mathbf{B}$, where $y=\mid\left\{\mathbf{B}_{i k} \mid\right.$ $\left.\mathbf{B}_{i k}=\mathbf{B}_{j k}, 1 \leq k \leq m\right\} \mid$ and $x=m-y$.

2. From 1., it follows that $y$ is the amount of equal elements between rows $i$ and $j$ in $\mathbf{B}$, and $x$ is the amount of elements that are unequal.

3. Let $\mathcal{B}$ the similarity matrix of $\mathbf{B}$ and $O$ the normalized matrix of $A=\mathcal{B}\left(\mathcal{B}^{T}\right)$ according to Definition 3.

4. From 3. and the definition of inner product multiplication, it follows that each cell of the matrix $A$ is calculated as follows: $A_{i j}=\sum_{k=1}^{m} \mathcal{B}_{i k} \mathcal{B}_{j k}$.

5. From Definition 2 it follows that if $\mathcal{B}_{i k}=\mathcal{B}_{j k}$, then $\mathcal{B}_{i k} \mathcal{B}_{j k}=1$, and otherwise $\mathcal{B}_{i k} \mathcal{B}_{j k}=-1$.

6. From 4. and 5., it follows that $\sum_{k=1}^{m} \mathcal{B}_{i k} \mathcal{B}_{j k}=y-x$. Therefore $A_{i j}=y-x$.

7. From 6. and Definition 3, it follows that $O_{i j}=\frac{A_{i j}+m}{2}=\frac{y-x+m}{2}=\frac{y-x+x+y}{2}=$ $y$.

8. From 2. and 7., it follows that $O_{i j}$ is the amount of equal elements between rows $i$ and $j$ in $\mathbf{B}$. 
Lemma 1. The Hamming distance between two ballots $B_{i}$ and $B_{j}$ is equal to $m-w_{i j}$ in the corresponding voter graph $G_{V}$, i.e. $H\left(B_{i}, B_{j}\right)=m-w_{i j}$.

Proof. We prove this lemma directly.

1. Suppose two ballots $B_{i}$ and $B_{j}$ containing $m$ issues, and $y$ to be the amount of issues on which the voters $i$ and $j$ agree.

2. From 1. and Theorem 1 it follows that the voter-to-voter normalised matrix $V$, constructed from a profile $\mathbf{B}$ containing $B_{i}$ and $B_{j}$, has $V_{i j}=y$.

3. From 2. and the construction of the voter-to-voter matrix, it follows that $G_{V}$ is the voter graph constructed from $V$ and the weight of the edge between the vertices $i$ and $j$ in $G_{V}$, written $w_{i j}$, is $y$.

4. From 3. and Hamming distance definition, it follows that the Hamming distance $H\left(B_{i}, B_{j}\right)=m-y$,

5. From 4. and 2., it follows that $H\left(B_{i}, B_{j}\right)=m-w_{i j}$.

Lemma 2. The Hamming distance between a ballot $B_{i}$ and a profile $\boldsymbol{B}$ is equal to $m n-s_{i}$, where $s_{i}$ is the sum of the weights of the incident edges of vertex $i$ in the voter graph constructed from $\boldsymbol{B}$ :

$$
H\left(B_{i}, \boldsymbol{B}\right)=m n-s_{i}
$$

Proof. Suppose some profile $\mathbf{B}$, a ballot $B_{i} \in \mathbf{B}$ and a voter graph $G_{V}$ constructed from $\mathbf{B}$. The Hamming distance between $B_{i}$ and $\mathbf{B}$ is

$$
\begin{aligned}
& \sum_{j \in \mathcal{N}} H\left(B_{i}, B_{j}\right) \\
= & \sum_{j \in \mathcal{N}} m-w_{i j} \\
= & m n-\sum_{j \in \mathcal{N}} w_{i j} \\
= & m n-s_{i}
\end{aligned}
$$

Lemma 3. The average voter rule AVR (Definition 1) selects the voters corresponding to the maximum total weight vertices in the voter graph, i.e.:

$$
A V R(\boldsymbol{B})=\underset{i \in V_{V}}{\operatorname{argmax}} s_{i} .
$$

Proof.

$$
\begin{aligned}
A V R(\mathbf{B}) & =\underset{B \in \operatorname{Supp}(B)}{\operatorname{argmin}} \mathcal{H}(B, \mathbf{B}) \\
& =\underset{i \in V_{V}}{\operatorname{argmin}}\left(m n-s_{i}\right) \\
& =\underset{i \in V_{V}}{\operatorname{argmax}} s_{i}
\end{aligned}
$$

